\title{
A Monte Carlo study of Electron Transport in Strained Si/SiGe Heterostructures
}

\author{
MAHBUB RASHED ${ }^{a}$, W.-K. SHIH ${ }^{a}$, S. JALLEPALLI ${ }^{a}$, R. ZAMAN ${ }^{a}$, T. J. T. KWAN ${ }^{b}$ and C. M. MAZIAR ${ }^{a}$ \\ ${ }^{a}$ Microelectronics Research Center, The University of Texas at Austin \\ ${ }^{b}$ Los Alamos National Laboratory, New Mexico
}

\begin{abstract}
Electron transport in pseudomorphically-grown silicon on relaxed (001) $\mathrm{Si}_{1-\mathrm{x}} \mathrm{Ge}_{\mathrm{x}}$ is investigated using a Monte Carlo (MC) simulation tool. The study includes both electron transport in bulk materials and in nMOS structures. The bulk MC simulator is based on a multiband analytical model, "fitted bands", representing the features of a realistic energy bandstructure. The investigation includes the study of low- and high-field electron transport characteristics at $77 \mathrm{~K}$ and $300 \mathrm{~K}$. Single particle MC simulations are performed for a strained silicon nMOS structure at room temperature. Both calculations show saturation of mobility enhancement in strained silicon beyond germanium mole fraction of 0.2 .
\end{abstract}

\section{INTRODUCTION}

The scaling of silicon MOSFET channel lengths to increase functional density, speed and drive current has pushed the limit of gate length towards $0.1 \mu \mathrm{m}$. An alternate approach to enhance drive current is through increasing the mobility of the charge carriers, that is the electrons or holes in the channel. Recent reports have shown that electron mobility is enhanced when electrons flow in a strained silicon channel pseudomorphically grown on relaxed $\mathrm{Si}_{1-\mathrm{x}} \mathrm{Ge}_{\mathrm{x}}$ [1-4]. These demonstrations of higher mobility hold significant promise for silicon based heterostructure devices in high speed digital and analog applications. When silicon epi-layers are pseudomorphically grown on a (001) $\mathrm{Si}_{1-\mathrm{x}} \mathrm{Ge}_{\mathrm{x}}$ substrate, the epi-layers experience a biaxial in-plane extension. This tensile biaxial strain raises the energy of the four conduction band minima on the in-plane $<100>$ axes with respect to the two minima on the $<001>$ axes. The magnitude of separa- tion of the two sets of valley minima has been measured to be $\Delta E \approx 0.67 \times x \mathrm{eV}$, where $\mathrm{x}$ is the Ge mole fraction [2]. This results in a redistribution of the electrons between the two sets of valleys, with more electrons populating the lower valleys, where they exhibit a lower effective mass for transport. The enhancement of mobility is due to the realization of lower effective mass transport and the suppression of intervalley scattering because of the lifting of the degeneracy. In this paper, we report a detailed investigation of the electron transport in strained silicon using a fittedband MC code [5] for bulk simulation and a MC tool that includes channel quantization effects in nMOS structures. Single particle simulation results are presented for both $300 \mathrm{~K}$ and $77 \mathrm{~K}$. In the case of nMOS structures, a single particle MC simulation is performed by solving a two-dimensional multi-subband Boltzmann Transport Equation (BTE). Effective mobility enhancement in strained-silicon nMOS is compared with recent experimental results. 


\section{MODELS}

As stated earlier, the tensile biaxial strain splits the conduction bands along A. The bands along [001] and $[00-\overline{1}]$ are lowered in energy with respect to the mean energy while bands along [100], [100], [010] and [010] are raised in energy. The bands along $\mathrm{L}$ are unaffected since there is no strain tensor along this direction. The MC simulator for silicon, SLAPSHOT [5], has been modified and enhanced to investigate electron transport in strained-Si (001) layers grown on relaxed (001) substrates. The scattering rate computation is based on a nonlocal pseudopotential band structure. The effective masses and nonparabolicities of different valleys are assumed to be unaffected by the strain [2]. Changes of the phonon coupling constants for the various scattering modes due to strain and other second-order effects are ignored. Acoustic intravalley and intervalley phonon scattering, optical phonon scattering, ionized impurity scattering and carrier-carrier scattering are included. Ionized impurity scattering is taken into account for a background doping concentrations of $10^{14} \mathrm{~cm}^{-3}$ and an electron density of $10^{18} \mathrm{~cm}^{-3}$ is used to approximate the sheet charge of $10^{12} \mathrm{~cm}^{-2}$ in a $10 \mathrm{~nm}$ modulation doped channel.

In the second part of the work, where the quantization effects are included, the subband structure is calculated by applying a formalism based on the effective-mass approximation with a bulk non-parabolic $\mathrm{E}(\mathbf{K})$ relation, as described in detail in [6] for the case of silicon MOSFETs. In the $\mathrm{Si} / \mathrm{SiGe}$ system of interest, the following zeroth-order effective-mass equation applies :

$$
\begin{aligned}
& {\left[-\frac{\hbar^{2}}{2 \mathrm{~m}_{\mathrm{z}}^{(\mathrm{i})}} \frac{\partial^{2}}{\partial \mathrm{z}^{2}}+\mathrm{E}_{\mathrm{c}}^{(\mathrm{i})}(\mathrm{z})+\gamma_{2 \mathrm{D}}^{(\mathrm{i})}\left(\mathrm{k}_{\mathrm{x}}, \mathrm{k}_{\mathrm{y}}\right)\right] \zeta(\mathrm{z})=\mathrm{E} \zeta(\mathrm{z}),} \\
& \gamma_{2 \mathrm{D}}^{(\mathrm{i})}\left(\mathrm{k}_{\mathrm{x}}, \mathrm{k}_{\mathrm{y}}\right)=\frac{\hbar^{2} \mathrm{k}_{\mathrm{x}}^{2}}{2 \mathrm{~m}_{\mathrm{x}}^{(\mathrm{i})}}+\frac{\hbar^{2} \mathrm{k}_{\mathrm{y}}^{2}}{2 \mathrm{~m}_{\mathrm{y}}^{(\mathrm{i})}}
\end{aligned}
$$

It is readily seen that using identical effective masses for $\mathrm{Si}$ and $\mathrm{SiGe}$ allows $\gamma 2 \mathrm{D}$ to be dropped globally and renders $m_{\mathrm{z}} \mathrm{z}$-independent. As a result, the calculation of the subband dispersion relation in the $\mathrm{Si} /$ $\mathrm{SiGe}$ system is essentially the same as in silicon
nMOS except that the bulk conduction-band offset $\Delta \mathrm{E}$ at the $\mathrm{Si} / \mathrm{SiGe}$ interfaces must be included in the potential term $\mathrm{E}_{\mathrm{c}}(\zeta)$ in the final $1 \mathrm{D}$ effective-mass Schroedinger equation. As in the case of silicon, the resulting subbands can be classified into ladders according to the bulk valleys from which they originiate. For the strained channel, the values of the conduction band offsets were found to be $-0.45 \times x \mathrm{eV}$ for the twofold out-of-plane bands and $0.22 \times x \mathrm{eV}$ for the fourfold in-plane bands[2]. The scattering of the 2D electrons due to bulk phonons and surface roughness is included. In the phonon scattering rate calculation, we have adopted the formalism developed by Price [7]. Scatterings due to ionized impurities, being important only in the presence of large interface charge density or when the channel is weakly inverted, are ignored. Surface roughness scattering is modeled with a simple formalism introduced by Cheng [8].

In the case under study, a long-channel strained-silicon nMOS structure with uniform substrate doping concentrations and zero source-drain bias is simulated. For a given substrate doping concentrations, the gate voltage is ramped from weak inversion to the strong inversion regime. At each gate bias, a two dimensional potential profile for the device is obtained by solving the drift-diffusion (DD) and Poisson equations using the DD formalism in UT-MINIMOS [10]. Since a uniform inversion layer is assumed, a slice along the depth is selected at the middle of the channel. One dimensional Schroedinger and Poisson equations are then solved simultaneously for the slice in order to calculate subband structures. This is followed by a single particle MC simulation, where a single particle is launched according to the density -of-states (DOS) weighted equilibrium distribution. An adequate number of subbands (fifteen subbands in this study) and an upper energy boundary are chosen in order to avoid running out of DOS in the simulation. The Pauli exclusion principle is included in the region of ohmic transport, where scattering rates are reduced due to the reduced availability of final states. 


\section{RESULTS OF THE SIMULATION}

The results presented here were obtained from simulations for fixed fields at $77 \mathrm{~K}$ and $300 \mathrm{~K}$ for the bulk case. The fields were applied along the $<100>$ and $<001>$ directions. The mobility of the electrons in the strained-silicon depends on the direction of the field due to the valley repopulation. When the field is applied along the $\langle 100\rangle$ direction, the lighter transverse mass transport is realized. On the other hand when the field is applied along $<001>$, the heavier longitudinal mass reduces the electron mobility. The mobility for the field along $<001>$ is even lower than that of unstrained silicon due to the heavier-effectivemass transport. Since fields along $<100>$ offer superior transport characteristics, we have chosen to compare the results of transport simulations for strained and unstrained silicon along this direction. Fig. 1 dis-

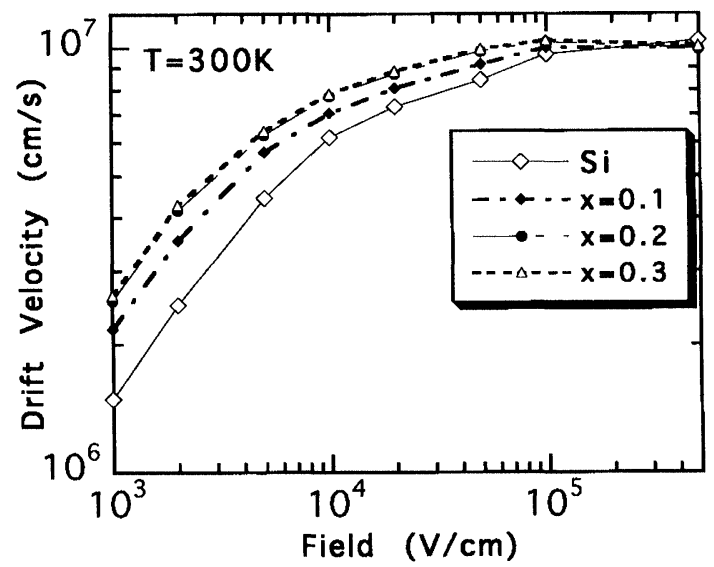

FIGURE 1 Velocity vs. field characteristics of strained $\mathrm{Si}$ on $\mathrm{Si}_{1-}$ ${ }_{x} \mathrm{Ge}_{\mathrm{x}}$ for different germanium mole fractions at $300 \mathrm{~K}$. Mobility enhancement saturates beyond $\mathrm{x}=0.2$

plays the velocity-field characteristics at $300 \mathrm{~K}$ for several mole fractions of relaxed $\mathrm{Si}_{1-\mathrm{x}} \mathrm{Ge}_{\mathrm{x}}$. Mobility enhancement saturates as the mole fraction increases. This can be explained by the fact that with the increased Ge mole fraction more and more electrons reside in the two lowered valleys. This valley repopulation results in almost $100 \%$ of the population of electrons being located at $x=0.2$ where $\Delta \mathrm{E} \sim 0.134$ $\mathrm{eV}$. Further splitting has very little effect on the enhancement of mobility. The low-field mobility is about $2640 \mathrm{~cm}^{2} / \mathrm{Vs}$ at a Ge mole fraction of 0.3 as compared with that of unstrained silicon, $1450 \mathrm{~cm}^{2} /$ Vs. Recently reported room temperature Hall mobility has achieved the record high result of $2700 \mathrm{~cm}^{2} /$ V.s for a Ge mole fraction of 0.3 [2]. The velocityfield characteristics for several Ge mole fractions at $77 \mathrm{~K}$ are shown in Fig. 2. The low-field mobility is

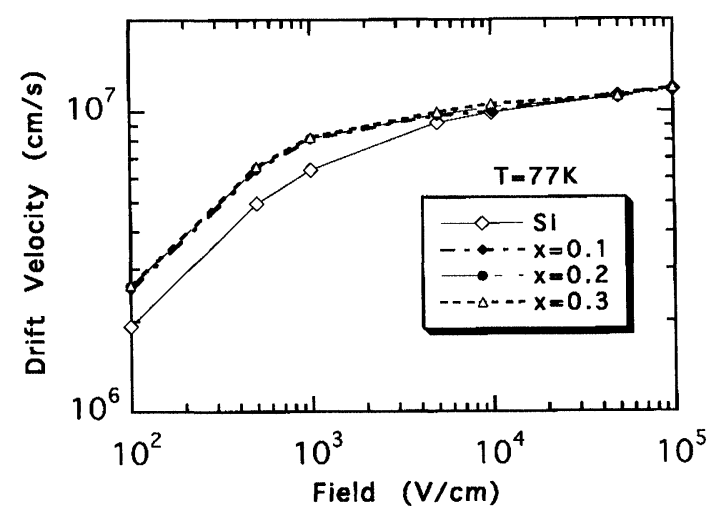

FIGURE 2 Velocity vs. field characteristics of strained $\mathrm{Si}$ on $\mathrm{Si}_{1-}$ ${ }_{\mathrm{x}} \mathrm{Ge}_{\mathrm{x}}$ for different strain conditions at $77 \mathrm{~K}$. Mobility enhancement saturates beyond $x=0.1$

found to be about $26000 \mathrm{~cm}^{2} / \mathrm{V} \cdot \mathrm{s}$ for strained silicon and $19000 \mathrm{~cm}^{2} / \mathrm{V} \cdot \mathrm{s}$ for unstrained intrinsic silicon. The low-field mobility in strained silicon is about 1.4 times that of unstrained intrinsic silicon. This corresponds to the ratio of conductivity effective masses of strained and unstrained silicon. This result is in good agreement with [2]. At $77 \mathrm{~K}$, the suppression of intervalley phonons becomes insignificant due to the sharp reduction of intervalley phonon scattering at such low temperatures, hence the enhancement is lower as compared to the $300 \mathrm{~K}$ case. As fields increase, electrons are distributed over the entire BZ and the curves merge. The saturation velocity obtained in our simulations is about $1.2 \times 10^{7} \mathrm{~cm} / \mathrm{s}$ at $77 \mathrm{~K}$.

The MC simulations for the nMOS structure were performed at room temperature. The enhancement of electron effective mobility in strained silicon channel nMOS structures as compared to conventional silicon nMOSFETs is presented for different strain conditions in Figure 3. This result compares well with the 


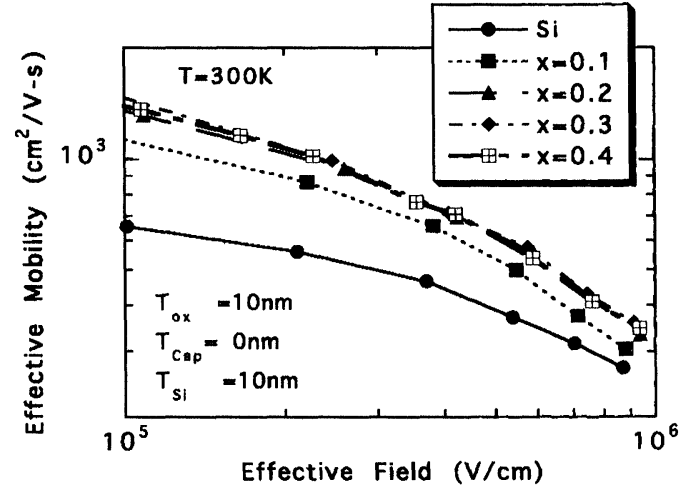

FIGURE 3 Effective mobility as a function of effective field for different strain conditions in a strained silicon nMOSFET. Significant enhancement of mobility is observed over a wide range of transverse fields

experimentally observed mobility enhancement that shows the saturation of mobility enhancement beyond germanium mole fractions of 0.2 [9].

\section{CONCLUSIONS}

In this present work, the electron transport in strained silicon pseudomorphically grown on relaxed $\mathrm{Si}_{1-\mathrm{x}} \mathrm{Ge}_{\mathrm{x}}$ (001) is explored using a MC tool. The drift velocity is calculated for different amounts of strain at $300 \mathrm{~K}$ and $77 \mathrm{~K}$ using a bulk MC simulator. Superior transport characteristics are simulated for strained silicon along $\langle 100\rangle$ as compared to unstrained silicon This is because of the reduction of intervalley phonon scattering due to the splitting of the six-fold degeneracy of the bands and to transport characterized by the lighter transverse mass. Low field mobility shows significant enhancement for the strained case, however, only a slight increase in the saturation velocity is observed. Effective electron mobility in strained layer nMOS structure has also been studied for different strained conditions. These tools will be used in future work to design and analyze strained $\mathrm{Si} / \mathrm{Si}_{1-\mathrm{x}} \mathrm{Ge}_{\mathrm{x}}$ devices.

\section{Acknowledgements}

This work was sponsored, in part, under the Joint Services Electronics Program, Contract Number F49620-95-C-0045, by the Semiconductor Research Corporation, by Motorola and by the Texas Advanced Technology Program. The United States Government is authorized to reproduce and distribute reprints for governmental purposes notwithstanding any copyright notation hereon.

\section{References}

[1] K. Ismail, B. S. Meyerson, and P. J. Wang, Appl. Phys. Lett., vol. 58(19), pp. 2117-2119, 1991.

[2] Th. Vogelsang and K. R. Hofmann, Appl. Phys. Lett., vol.63(2), pp. 186-188, 1993.

[3] H. Miyata, T. Yamada, and D. K. Ferry, Appl. Phys. Lett., vol.62(21), pp. 2661-2663, 1993.

[4] A. Abramo, J. Bude, F. Venturi, and M. Pinto, IEDM, pp-, 1994.

[5] X. L. Wang, V. Chandramouli, C. M. Maziar, and A. F. Tasch, J. Appl. Phys., vol. 73, no. 7, pp. 3339-3347, 1993.

[6] W. -K. Shih, S. Jallepalli, C. -F. Yeap, M. Rashed, C. M. Maziar, and A. F. Tasch Jr., Proceedings of IEEE UGIM Symposium, Austin, May 17-18, 1995.

[7] P. J. Price, Anls. Phys. vol.133, pp. 217, 1981.

[8] Y. C. Cheng, Surf Sci., vol. 27, p. 663, 1971.

[9] J. Welser, J. L. Hoyt, S. Takagi, and J. F. Gibbons, pp. 373376, IEDM-1994.

[10] Choi-Fei Yeap, Ph.D Dissertation, UT-Austin, 1995. 

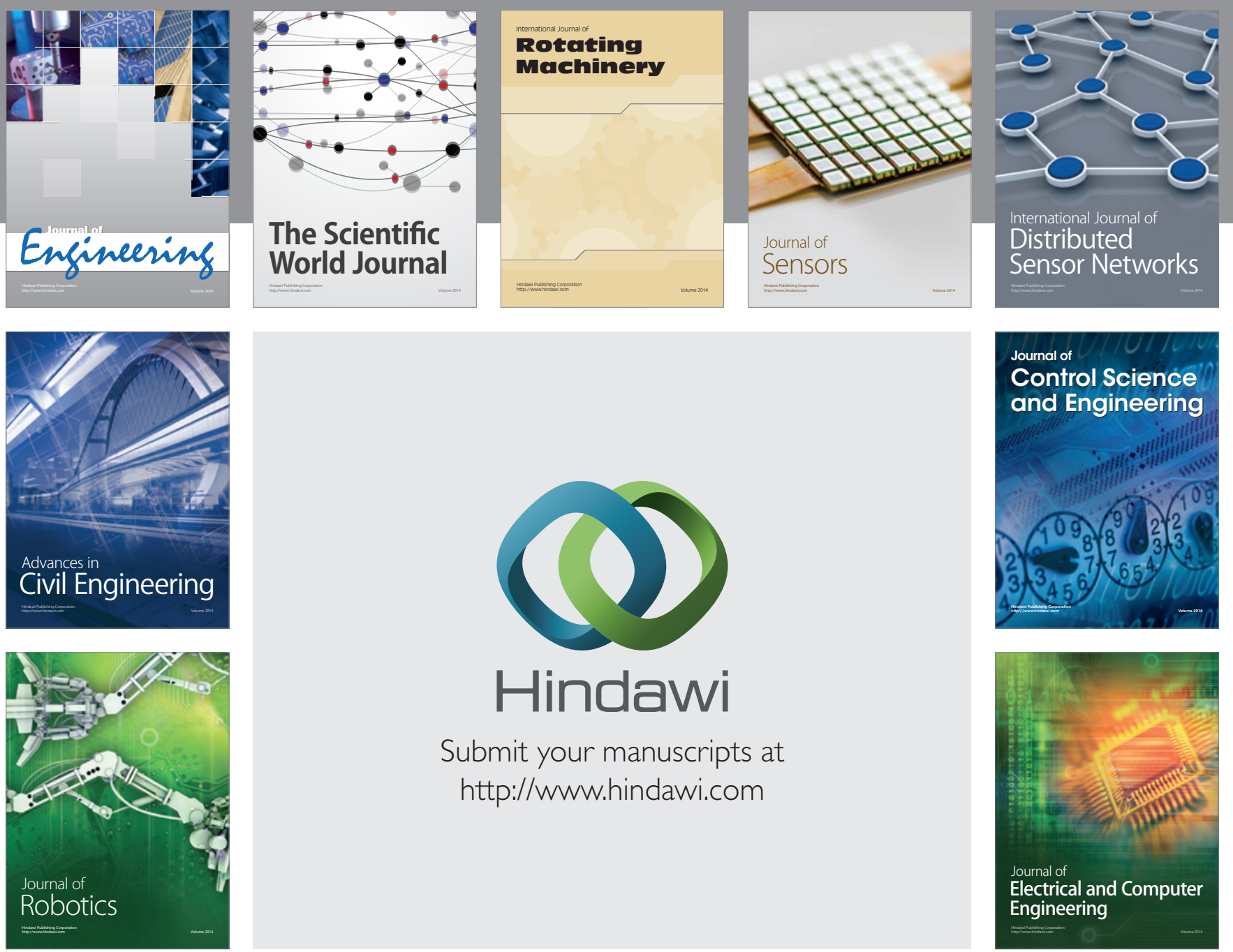

Submit your manuscripts at

http://www.hindawi.com
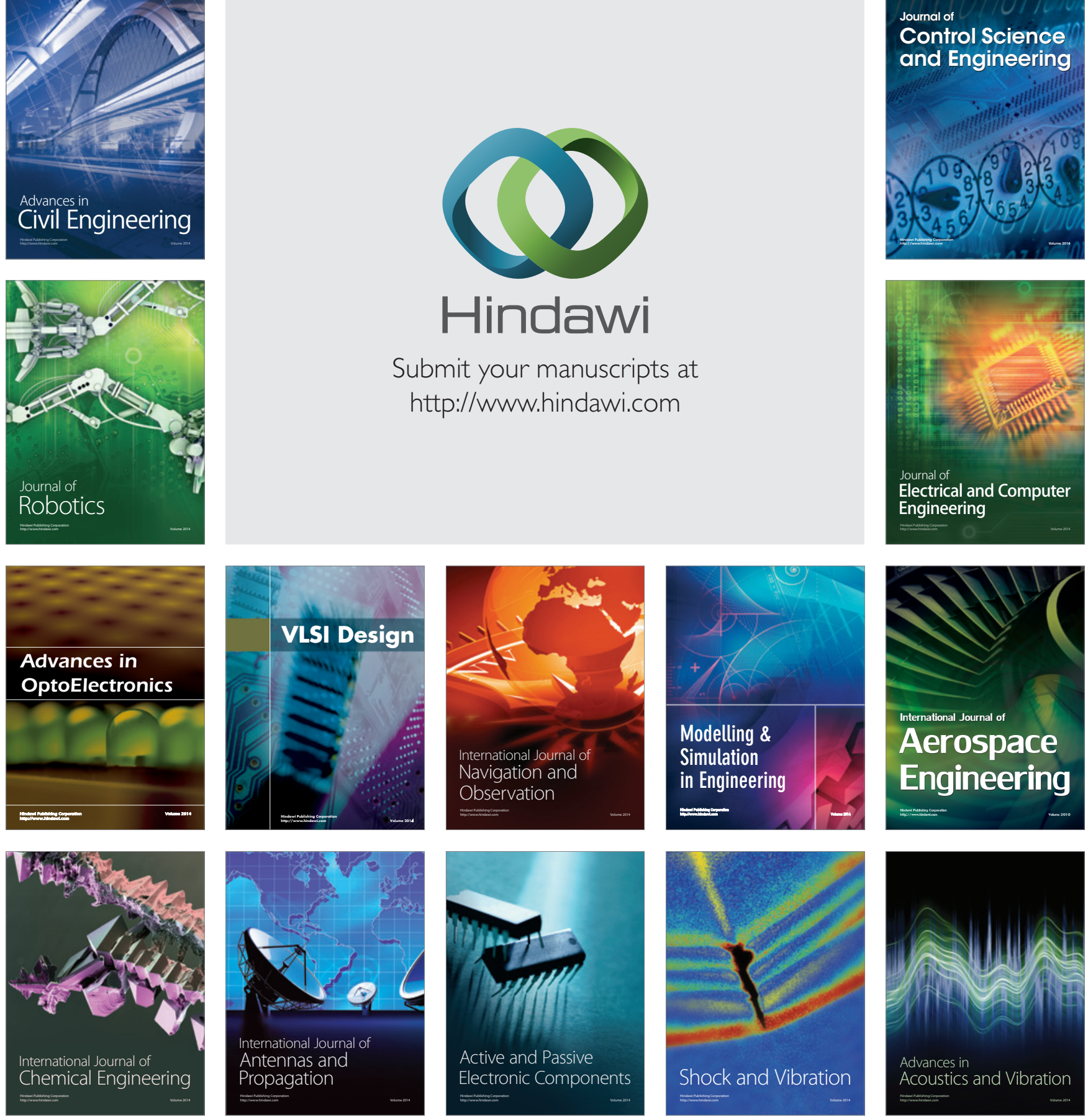